\title{
SPECTRUM OF A SPECTRAL OPERATOR ${ }^{1}$
}

KIRTI K. OBERAI

1. Preliminaries. Foguel [2] proved that if $T$ is a spectral operator on a Banach space then the spectrum of $T$ coincides with its approximate spectrum. In this paper we show that this is not necessarily so on a locally convex space and we obtain the conditions under which the result is true.

Throughout this paper $E$ will denote a separated, complex locally convex space which we shall assume to be quasi-complete and barreled. Let $E^{\prime}$ be the dual of $E$ and let $\mathcal{L}(E)$ be the algebra of all continuous linear maps of $E$ into itself. Let $I$ be the identity map of $E$ on to itself. On $\mathscr{L}(E)$ we shall always consider the topology $\tau$ of uniform convergence on the bounded subsets of $E$. Also, let $\Gamma$ be the class of Borel subsets of the complex plane $C$ and let $\hat{C}$ be one point compactification of $C$.

On a locally convex space the spectrum of an operator $T \in \mathscr{L}(E)$ is defined in the sense of Waelbroeck [5] as follows:

$\lambda \in \hat{C}$ is said to belong to the resolvent set $\rho(T)$ of $T$ if and only if there is a neighborhood $V_{\lambda}$ of $\lambda$ in $\hat{C}$ such that there is a function $\mu \rightarrow R_{\mu}$ on $V_{\lambda} \cap C$ to $\mathscr{L}(E)$ satisfying, for each $\mu \in V_{\lambda} \cap C$, the conditions

(i) $R_{\mu}(\mu I-T)=(\mu I-T) R_{\mu}=I$;

(ii) $\left\{R_{\mu}: \mu \in V_{\lambda} \cap C\right\}$ is bounded in $\mathscr{L}(E)$.

The spectrum, $\operatorname{sp}(T)$, of $T$ is defined by $\operatorname{sp}(T)=\hat{C}-\rho(T)$. If $\infty \notin \operatorname{sp}(T)$ then $T$ is called a regular element of $\mathcal{L}(E)$.

The following is the classical definition of spectrum of $T$.

$\lambda \in C$ is said to belong to the resolvent set $\rho^{c}(T)$ of $T \in \mathcal{L}(E)$ if and only if $(\lambda I-T)^{-1}$ exists as an everywhere defined continuous operator on $E$. The spectrum, $\operatorname{sp}^{c}(T)$ of $T$ is defined by

$$
\operatorname{sp}^{c}(T)=C-\rho^{c}(T) .
$$

The two definitions are equivalent on a Banach space. However, Maeda [4] gave examples to show that the two definitions are not necessarily equivalent even on a Fréchet space. We shall call an operator $T \in \mathscr{L}(E)$ an operator of type [B] if the above definitions of the spectrum of $T$ are equivalent. The examples of operators of type

Received by the editors July 18, 1966.

1 This paper is based on author's dissertation submitted to the University of Illinois for the $\mathrm{Ph} . \mathrm{D}$. degree. The author wishes to express his gratitude to Professor R. G. Bartle for his valuable suggestions and guidance. Also, this research was partially supported by the NSF. 
[B] are completely bounded operators and compact operators [4]. We now give a few definitions. Most of these are based on [3]. A function $\{P(\sigma): \sigma \in \Gamma\}$ of commuting projection valued operators defined on $\Gamma$ with values in $\mathscr{L}(E)$ is called a spectral measure on $E$ if

(a) for each $x \in E, P(\cdot) x$ is countably additive in $E$;

(b) $P(C)=I$;

(c) $\{P(\sigma): \sigma \in \Gamma\}$ is an equicontinuous part of $\mathscr{L}(E)$.

(Under the assumption that $E$ is barreled condition (c) may be deduced from (a).)

$T \in \mathfrak{L}(E)$ is called a spectral operator on $E$ if there exists a (unique) spectral measure $P(\cdot)$ on $E$ such that

(a) $T P(\sigma)=P(\sigma) T$, for all $\sigma \in \Gamma$;

(b) $\operatorname{sp}\left(T_{\sigma}\right) \subset \bar{\sigma}, \sigma \in \Gamma$ where $T_{\sigma}$ is the restriction of $T$ to $P(\sigma) E$;

(c) for each $x \in E$ and $x^{\prime} \in E^{\prime}$, the complex measure $\left\langle P(\cdot) x, x^{\prime}\right\rangle$ has compact support in $C$.

$S \in \mathscr{L}(E)$ is called a scalar operator on $E$ if there exists a (unique) spectral measure $P(\cdot)$ on $E$ such that for each $x \in E$ and $x^{\prime} \in E^{\prime}, \lambda$ is integrable with respect to $\left\langle P(\cdot) x, x^{\prime}\right\rangle$ and

$$
\left\langle S x, x^{\prime}\right\rangle=\int \lambda d\left\langle P(\lambda) x, x^{\prime}\right\rangle .
$$

$N \in \mathfrak{L}(E)$ is called quasi-nilpotent if for each $x \in E$ and $x^{\prime} \in E^{\prime}$, $\lim _{n \rightarrow \infty}\left|\left\langle N^{n}, x, x^{\prime}\right\rangle\right|^{1 / n}=0$.

If the spectral measure $P(\cdot)$ corresponding to a spectral operator $T$ satisfies the condition $P C_{0}$ (refer to [3]) then $T$ has a unique (canonical) decomposition $T=S+N$ where $S$ is a scalar operator having the same spectral measure as $T$ and $N$ is a quasi-nilpotent operator commuting with $S$. $S$ is called the scalar part of $T$ and $N$ the radical of $T$. In the sequel, whenever we consider a spectral operator $T, S, N$ and $P(\cdot)$, respectively, will denote the scalar part, the radical and the spectral measure corresponding to $T$. Also $T$ is said to be of finite type if $N^{n}=0$ for some positive integer $n$.

Lemma 1. $A$ net $\left(T_{\alpha}\right)$ in $\mathfrak{L}(E)$ converges to $T \in \mathfrak{L}(E)$ in the topology $\tau$ if and only if for each bounded set $B$ in $E$ and each equicontinuous set $A$ in $E^{\prime}$

$$
\left\langle T_{\alpha} x, x^{\prime}\right\rangle \rightarrow\left\langle T x, x^{\prime}\right\rangle, \quad x \in B, x^{\prime} \in A
$$

uniformly on $B$ and $A$.

Proof. The topology on $E$ is the topology of uniform convergence on the equicontinuous subsets of $E^{\prime}$. Hence $\left\langle T_{\alpha} x, x^{\prime}\right\rangle \rightarrow\left\langle T x, x^{\prime}\right\rangle$ uni- 
formly on $B$ and $A$ if and only if $T_{\alpha} x \rightarrow T x$ uniformly on $B$, which is true if and only if $T_{\alpha} \rightarrow T$ in $\tau$.

Definition 1. A spectral measure $P(\cdot)$ on $E$ is said to satisfy the compactness condition if for each bounded set $B$ in $E$ and each equicontinuous set $A$ in $E^{\prime}$ there exists a compact set $\sigma(A, B)$ in $C$ such that

$$
\operatorname{Supp}\left\langle P(\cdot) x, x^{\prime}\right\rangle \subset \sigma(A, B) \text { for all } x \in B, x^{\prime} \in A \text {. }
$$

If $T$ is a spectral operator and $P(\cdot)$ satisfies the compactness condition, then we say that $T$ satisfies the compactness condition. If the spectrum of $T$ is compact then $T$ automatically satisfies the compactness condition. It may be remarked that in this case $P(\cdot)$ also satisfies the condition $P C_{0}$.

Lemma 2. Let $P(\cdot)$ be a spectral measure on $E$ satisfying the compactness condition. Then $\int \lambda d P(\lambda)$ exists as a Riemann-Stieltjes integral in the topology $\tau$.

Proof. Let $B$, a bounded set in $E$, and $A$, an equicontinuous part in $E^{\prime}$, be fixed. Let $\sigma$ be a compact set in $C$ such that

$$
\operatorname{Supp}\left\langle P(\cdot) x, x^{\prime}\right\rangle \subset \sigma \quad \text { for all } x \in B \quad \text { and } \quad x^{\prime} \in A .
$$

Let $\left(\sigma_{i}\right)_{i=1}^{n}$ and $\left(\delta_{j}\right)_{j=1}^{m}$ be any two subdivisions of $\sigma$ into disjoint Borel sets of diameters less than any given $\epsilon>0$. If $\alpha_{i} \in \sigma_{i}$ and $\beta_{j} \in \delta_{j}$ then

$$
\begin{aligned}
\sup _{x \in B ; x^{\prime} \in A} & \left\langle\sum_{i} \alpha_{i} P\left(\sigma_{i}\right) x, x^{\prime}\right\rangle-\left\langle\sum_{j} \beta_{j} P\left(\delta_{j}\right) x, x^{\prime}\right\rangle \mid \\
& =\sup _{x \in B ; x^{\prime} \in A}\left|\left\langle\sum_{i, j}\left(\alpha_{i}-\beta_{j}\right) P\left(\sigma_{i} \cap \delta_{j}\right) x, x^{\prime}\right\rangle\right| \\
& <2 \epsilon \cdot 4 K
\end{aligned}
$$

where $K=\sup \left|\left\langle P(\sigma) x, x^{\prime}\right\rangle\right|$, the supremum being taken over all $\sigma \in \Gamma, x \in B$ and $x^{\prime} \in A . K$ is finite since $\{P(\sigma): \sigma \in \Gamma\}$ is an equicontinuous part of $\mathscr{L}(E)$. The lemma now follows from the definition of the integral and Lemma 1.

CoROLlary 1. If $S$ is a scalar operator which satisfies the compactness condition, then

$$
S=\int \lambda d P(\lambda) .
$$

The integral exists in the topology $\tau$. 
THEOREM 1. Let $T$ be a spectral operator on $E$ satisfying the compactness condition. Let $P(\{0\})=0$. Then $(T E)^{-}=E$.

The proof of this theorem may be obtained in a series of lemmas including Lemma 2. Since the proof is very similar to [2, Corollary 2] we omit the details for which the reader may refer to author's dissertation.

Lemma 3. Let $T$ be as in Theorem 1. Let $\sigma$ be a closed set in $C$ and let $\lambda \notin \sigma$. Also, let $(\lambda I-T) x=0$ for some $x \in E$. Then $P(\sigma) x=0$ and $P(\{\lambda\}) x=x$.

Proof. Since $\lambda \notin \sigma=\bar{\sigma}, \lambda \notin \operatorname{sp}\left(T_{\sigma}\right)$. Therefore, $\left(\lambda I-T_{\sigma}\right)^{-1}$ exists as an everywhere defined continuous operator on $P(\sigma) E$ so that

$$
(\lambda I-T)_{\sigma}^{-1}(\lambda I-T) P(\sigma)=P(\sigma) .
$$

Now

$$
P(\sigma) x=(\lambda I-T)_{\sigma}^{-1} P(\sigma)(\lambda I-T) x=0 .
$$

Also

$$
P(\{\lambda\}) x+\lim _{n \rightarrow \infty} P(\{z:|z-\lambda| \geqq 1 / n\}) x=P(C) x=x .
$$

Therefore, $P(\{\lambda\}) x=x$.

Corollary 2. If $P(\{0\})=0$ then $T$ is injective.

Proof. Suppose $T x=0$. Let $\sigma$ be any closed set in $C$ such that $0 \notin \sigma$. By taking $\lambda=0$ in the above lemma we have,

$$
0=P(\{0\}) x=x .
$$

2. Classification of spectrum of $T$. In this section we shall always assume that $T$ is an operator of type [B] and that $E$ is a locally convex space in which the closed graph theorem holds in the sense that any closed linear operator defined on all of $E$ is continuous. Also, any spectral operator on $E$ will be assumed to satisfy the compactness condition. The spectrum of any $T \in \mathcal{L}(E)$ is classified as follows:

1. The point spectrum, $\operatorname{sp}_{p}(T)$, of $T$ is the set of all $\lambda \in C$ such that $(\lambda I-T)$ is not injective on $E$ so that $(\lambda I-T)^{-1}$ does not exist.

2. The continuous spectrum, $\operatorname{sp}_{c}(T)$, of $T$ is the set of all $\lambda \in C$ such that $(\lambda I-T)$ is injective on $E$ and the range, $R(\lambda I-T)$, of $(\lambda I-T)$ is a proper dense subset of $E$.

3. The residual spectrum, $\operatorname{sp}_{r}(T)$, of $T$ is the set of all $\lambda \in C$ such that $(\lambda I-T)$ is injective on $E$ and $R(\lambda I-T)$ is not dense in $E$. 
Since $T$ is an operator of type [B], from the closed graph theorem it follows that $\mathrm{sp}(T)=\mathrm{sp}_{p}(T) \cup \operatorname{sp}_{c}(T) \cup \operatorname{sp}_{r}(T)$, and the sets on the right-hand side are pairwise disjoint.

DeFinition 2. $\lambda \in C$ is said to belong to the approximate spectrum, $\operatorname{sp}_{a}(T)$, of $T$ if there exists a continuous seminorm $p$ on $E$ and a net $\left(x_{\alpha}\right)$ in $E$ such that $p\left(x_{\alpha}\right) \geqq 1$ for all $\alpha$ and such that for each continuous seminorm $q$ on $\left.E, q(\lambda I-T) x_{\alpha}\right) \rightarrow 0$.

It is clear that $\lambda \in \operatorname{sp}_{a}(T)$ if and only if there is a neighborhood $U$ of 0 in $E$ and a net $\left(x_{\alpha}\right) \subset E \sim U$ such that $(\lambda I-T) x_{\alpha} \rightarrow 0$ in $E$.

From the above definition, it is not hard to prove the following.

Lemma 4. (a) $\operatorname{sp}_{a}(T) \subset \mathrm{sp}(T) ;(\mathrm{b}) \mathrm{sp}_{p}(T) \subset \mathrm{sp}_{a}(T)$.

Proposition 1. Let $T$ be a spectral operator on $E$ and let $\lambda \in \operatorname{sp}(T)$. Then (a) if $P(\{\lambda\})=0$ then $\lambda \in \operatorname{sp}_{c}(T)$ (b) if $P(\{\lambda\}) \neq 0$ and $T$ is of finite type then $\lambda \in \operatorname{sp}_{p}(T)$.

Proof. (a) The operator $(\lambda I-T)$ is a spectral operator on $E$ whose spectral measure $Q(\cdot)$ is defined by $Q(\sigma)=P(\lambda-\sigma)$, so that $Q(\{0\})$ $=P(\{\lambda\})=0$. Hence by Theorem $1,((\lambda I-T) E)^{-}=E$. Also, by Corollary 2, $(\lambda I-T)$ is injective. Since $\lambda \notin \rho(T)$, it follows that $\lambda \in \operatorname{sp}_{c}(T)$.

(b) Let $0 \neq x \in P(\{\lambda\}) E$ so that $x=P(\{\lambda\}) x$. We have,

$$
\begin{aligned}
S x & =\int \mu d(P(\mu) x)=\int \mu d(P(\mu) P(\{\lambda\}) x)=\int \mu d(P(\mu \cap\{\lambda\}) x) \\
& =\lambda P(\{\lambda\}) P(\{\lambda\}) x=\lambda x .
\end{aligned}
$$

Since $T$ is of finite type, there is a positive integer $n$ such that $N^{n} x=0$ and $N^{n-1} x \neq 0$. Therefore, $T N^{n-1} x=(S+N) N^{n-1} x=\lambda N^{n-1} x$. Thus $\lambda \in \operatorname{sp}_{p}(T)$.

COROLlaRy 3. The residual spectrum of a spectral operator of finite type and, in particular, of a scalar operator is empty.

Proposition 2. Let $E$ be complete and let $\lambda \notin \operatorname{sp}_{a}(T)$. Then $R(\lambda I-T)$ is closed in $E$.

Proof. Let $\left(y_{\alpha}\right)_{\alpha \in D}$ be a net in $R(\lambda I-T)$ and let $y_{\alpha} \rightarrow y$ in $E$. To prove the proposition we have to show that $y \in R(\lambda I-T)$.

There exist elements $z_{\alpha}$ in $E$ such that $(\lambda I-T) z_{\alpha}=y_{\alpha}$, for all $\alpha \in D$. We assert that $\left(z_{\alpha}\right)$ is a Cauchy net in $E$. Suppose this is not true so that there exists a neighborhood $U$ of 0 in $E$, such that for each $\alpha \in D$ there exist $\alpha^{\prime} \geqq \alpha$ and $\alpha^{\prime \prime} \geqq \alpha$ in $D$ such that $\left(z_{\alpha^{\prime}}-z_{\alpha^{\prime \prime}}\right) \notin U$. Consider the net $\left(z_{\alpha^{\prime}}-z_{\alpha^{\prime \prime}}\right)_{\alpha \in D}$. We have,

$$
(\lambda I-T)\left(z_{\alpha^{\prime}}-z_{\alpha^{\prime \prime}}\right)=y_{\alpha^{\prime}}-y_{\alpha^{\prime \prime}} .
$$


We shall show that $\left(y_{\alpha^{\prime}}-y_{\alpha^{\prime \prime}}\right)_{\alpha \in D} \rightarrow 0$. Let $V$ be any neighborhood of 0 in $E$. Let $W$ be a circled neighborhood of 0 in $E$ such that $W+W \subset V$. Since $y_{\alpha} \rightarrow y$ there is an $\alpha_{V} \in D$ such that $\left(y_{\alpha}-y\right) \in W$ for all $\alpha \geqq \alpha_{V}$. Let $\beta \geqq \alpha_{V}$ be arbitrary in $D$. Since $\beta^{\prime} \geqq \beta$ and $\beta^{\prime \prime} \geqq \beta$ it follows that $\beta^{\prime} \geqq \alpha_{V}$ and $\beta^{\prime \prime} \geqq \alpha_{V}$. Hence $\left(y_{\beta^{\prime}}-y\right) \in W$ and $\left(y_{\beta^{\prime \prime}}-y\right) \in W$ so that $y_{\beta^{\prime}}-y_{\beta^{\prime \prime}} \in W+W \subset V$, for all $\beta \geqq \alpha_{V}$. This implies that $\left(y_{\alpha^{\prime}}-y_{\alpha^{\prime \prime}}\right)_{\alpha \in D} \rightarrow 0$ in $E$. Thus, we have proved that $(\lambda I-T)\left(z_{\alpha^{\prime}}-z_{\alpha^{\prime \prime}}\right)$ $\rightarrow 0$ while $\left(z_{\alpha^{\prime}}-z_{\alpha^{\prime \prime}}\right) \cap U=\varnothing$. Therefore, by the definition of $\operatorname{sp}_{a}(T)$, $\lambda \in \operatorname{sp}_{a}(T)$ which is a contradiction. Hence, $\left(z_{\alpha}\right)$ is a Cauchy net and since $E$ is complete there is a $z \in E$ such that $z_{\alpha} \rightarrow z$; so that

$$
y_{\alpha}=(\lambda I-T) z_{\alpha} \rightarrow(\lambda I-T) z .
$$

Since $y_{\alpha} \rightarrow y$, and since $E$ is separated, it follows that $(\lambda I-T) z=y$. Thus, $y \in R(\lambda I-T)$. This proves the proposition.

Corollary 4. Let E be complete. Then $\operatorname{sp}_{c}(T) \subset \operatorname{sp}_{a}(T)$.

Proof. Let $\lambda \in \operatorname{sp}_{c}(T)$ so that $\lambda I-T$ is injective and $R(\lambda I-T)$ is dense in $E$. If $\lambda \notin \operatorname{sp}_{a}(T)$ then by the above proposition $R(\lambda I-T)$ is closed in $E$ and hence $R(\lambda I-T)=E$. By the closed graph theorem $\lambda \in \rho(T)$, which is a contradiction. Hence, $\lambda \in \operatorname{sp}_{a}(T)$.

THEOREM 2. Let $E$ be complete and let $T$ be a spectral operator of finite type on $E$. Then $\mathrm{sp}(T)=\mathrm{sp}_{a}(T)$.

Proof. By Corollary $3, \operatorname{sp}_{r}(T)=\varnothing$. Hence we have,

$$
\begin{aligned}
\operatorname{sp}_{a}(T) \subset \mathrm{sp}(T) & =\operatorname{sp}_{p}(T) \cup \mathrm{sp}_{c}(T) \\
& \subset \operatorname{sp}_{a}(T) \cup \mathrm{sp}_{a}(T) \\
& =\operatorname{sp}_{a}(T) .
\end{aligned}
$$

This implies that $\mathrm{sp}(T)=\mathrm{sp}_{a}(T)$.

Foguel [2] proved that if $T$ is a spectral operator on a Banach space then $\operatorname{sp}(T)=\operatorname{sp}_{a}(T)$. We give a simple example to show that this is not necessarily true even on a Fréchet space.

EXAmple. Consider the space $E$ of all sequences $\left(x_{n}\right)_{n=1}^{\infty}$ where all the $x$ 's are complex numbers. We define a countable family $p_{i}, i$ $=1,2, \cdots$, of seminorms on $E$ by

$$
p_{i}(x)=\sup _{k \leqq i}\left|x_{k}\right|,
$$

where $x=\left(x_{n}\right)$. This family defines a separated, locally convex topology $\tau$ on $E$ and $(E, \tau)$ is a Fréchet space.

Let $I$ be the identity operator on $E$ and let an operator $N$ on $E$ be defined by 


$$
N x=\left(0, x_{1}, x_{2}, \cdots\right) .
$$

Then $N$ is a quasi-nilpotent operator on $E$. Since $I$ is a scalar operator $T=I+N$ is a spectral operator on $E$. Now $1 \in \operatorname{sp}(I)=\operatorname{sp}(T)$. We assert that $1 \notin \operatorname{sp}_{a}(T)$. For, if $1 \in \operatorname{sp}_{a}(T)$ then there is a sequence $\left(x^{k}\right)$ in $E$ such that there is a neighborhood $U$ of 0 in $E$ such that $x^{k} \notin U$ for all $k$ and such that $(1 I-T) x^{k}=-N x^{k} \rightarrow 0$. This is not possible. Hence $\operatorname{sp}(T) \neq \operatorname{sp}_{a}(T)$.

\section{REFERENCES}

1. N. Dunford, Spectral operators, Pacific J. Math. 4 (1954), 321-354.

2. S. R. Foguel, The relation between a spectral operator and its scalar part, Pacific J. Math. 8 (1958), 51-65.

3. C. Ionescu Tulcea, Spectral operators on locally convex spaces, Bull. Amer. Math. Soc. 67 (1961), 125-128.

4. F. Maeda, Remarks on spectra of operators on a locally convex space, Proc. Nat. Acad. Sci. U.S.A. 47 (1961), 1052-1055.

5. L. Waelbroeck, Locally convex algebras, spectral theory, Seminar on complex analysis, Institute for Advanced Study, Princeton, N. J., 1958.

UNIVERSITY OF ILLINOIS AND

QUEEN'S UNIVERSITY 